\title{
Calibrated stellar models for metal-poor populations
}

\author{
P. Cariulo ${ }^{1}$, S. Degl'Innocenti ${ }^{1,2}$, and V. Castellani ${ }^{3,4}$ \\ ${ }^{1}$ Dipartimento di Fisica, Università di Pisa, via Buonarroti 2, 56126 Pisa, Italy \\ 2 INFN Sezione di Pisa, via Buonarroti 2, 56126 Pisa, Italy \\ 3 Osservatorio Astronomico di Roma, via Frascati 33, 00040 Monte Porzio Catone, Italy \\ ${ }^{4}$ INFN, sezione di Ferrara, via Paradiso 12, 44100 Ferrara, Italy
}

Received 16 June 2003 / Accepted 2 March 2004

\begin{abstract}
We extend to lower metallicities recent evolutionary computations devoted to Magellanic Clouds stars, presenting and discussing new stellar models with $Z=0.0002,0.0004,0.0006,0.001$ and suitable assumptions about the original He content. As in the previous paper, evolutionary results are compared with observational data to properly calibrate the assumptions about the efficiency of the surface convection. On this basis, we follow the evolution of stellar models in the mass range 0.6 to $11 M_{\odot}$ from the Main Sequence (MS) to the C ignition or the onset of thermal pulses in the advanced Asymptotic Giant Branch (AGB) phase, presenting cluster isochrones covering the range of ages from $20 \mathrm{Myr}$ to $20 \mathrm{Gyr}$. Selected predictions constraining the cluster ages are discussed, presenting a calibration of the difference in magnitude between the luminous MS termination and the He burning giants in terms of the cluster age. Both evolutionary tracks and isochrones are available at the URL http://astro.df.unipi.it/SAA/PEL/ZO.html ${ }^{\star}$.
\end{abstract}

Key words. stars: evolution - globular clusters: general - open clusters and associations: general

\section{Introduction}

In modern astrophysics, stellar clusters play the role of cosmic clocks marking the history of star populations in the Galaxy. Beyond the Galaxy, improved observational capability has already opened the study of stellar clusters in nearby galaxies, such as the Small and Large Magellanic Clouds, giving access to the history of star formation in the Universe. However, a precise determination of the ages of stellar clusters is still a problem addressed by several authors, investigating both the old Galactic globulars (see e.g., Chaboyer 2001; Salaris \& Weiss 2002) and the more recent disk population (see e.g., Carraro et al. 1999; Chaboyer et al. 1999). Thus, there is the need for extensive evolutionary calculations covering suitable grids of chemical compositions and ages.

In a previous paper (Castellani et al. 2003, Paper I) we presented evolutionary results for the Magellanic Cloud (LMC, SMC) chemical composition, prepared for the analysis of new HST observations of the intermediate-age LMC globular NGC 1866 (Brocato et al. 2003). The investigation of the deep color-magnitude $(\mathrm{CM})$ diagram of stellar populations in the Carina dwarf galaxy (Monelli et al. 2003) has recently suggested the extension of such evolutionary analysis to lower metallicities. In this paper we present these new results,

Send offprint requests to: S. Degl'Innocenti, e-mail: scilla@df.unipi.it

* Data files are available in electronic form at the CDS via anonymous ftp to cdsarc.u-strasbg.fr $(130.79 .128 .5)$ or via http://cdsweb.u-strasbg.fr/cgi-bin/qcat?J/A+A/421/1121 discussing the justification for several choices made when constructing these stellar models and comparing the results with similar computations available in the recent literature.

The reasons for presenting new theoretical computations have been discussed in Paper I, so they will only be stated briefly here. As a central point, the present set of models is currently the only one covering the phases of both $\mathrm{H}$ and He burning over a large range of masses with the inclusion of atomic diffusion of helium and heavy elements. This process has been demonstrated to sensitively affect the evolution of low mass stars, with consequences for the estimated ages of old clusters (see, e.g., Castellani \& Degl'Innocenti 1999; VandenBerg et al. 2002). We use throughout the canonical assumption of inefficient overshooting so the He burning structures are calculated according to the prescriptions of canonical semiconvection induced by the penetration of convective elements in the radiative region (Castellani et al. 1985). In the case of low mass stars this gives lifetimes for Horizontal Branch (HB) stars in better agreement with observational constraints than do overshooting models (see Paper I).

Since the occurrence and/or the amount of overshooting in more massive stars is still a debated question (see e.g. Bertelli et al. 2003, and references therein; Brocato et al. 2003), the availability of a set of canonical metal-poor models will be useful to further investigate this point. Moreover, as discussed in Appendix A, the present models are the only ones based on the most updated physics available in the literature. This does not necessarily imply that the updated physical inputs produce the most reliable models, but only that our models represent an 
Table 1. The three clusters selected for isochrone calibrations. For each cluster the columns give the source of photometry, the range of reddening values provided in the recent literature, $E(B-V)$, $[\mathrm{Fe} / \mathrm{H}]$ values as given by Zinn \& West (1984: ZW), Harris (2003: H) and Carretta \& Gratton (1997: CG), the amount of $\alpha$ enhancement from Salaris \& Cassisi (1996) and the estimates of the total metallicity, $Z$, as derived from $[\mathrm{Fe} / \mathrm{H}]$ values for the three quoted references.

\begin{tabular}{lccccccc}
\hline \hline Cluster & Photometry & $E(B-V)$ & {$[\mathrm{Fe} / \mathrm{H}]_{\mathrm{ZW}}$} & {$[\mathrm{Fe} / \mathrm{H}]_{\mathrm{H}}$} & {$[\mathrm{Fe} / \mathrm{H}]_{\mathrm{CG}}$} & {$[\alpha / \mathrm{Fe}]$} & $Z$ \\
\hline NGC 4590 (M 68) & Walker (1994) & $0.04 \div 0.09$ & $-2.09 \pm 0.11$ & -2.06 & $-1.99 \pm 0.10$ & 0.20 & $2.0 ; 2.1 ; 2.5 \times 10^{-4}$ \\
NGC 5272 (M 3) & Rey et al. (2001) & $0.01 \div 0.03$ & $-1.66 \pm 0.06$ & -1.57 & $-1.34 \pm 0.06$ & 0.26 & $5.9 ; 7.3 ; 12.3 \times 10^{-4}$ \\
NGC 6205 (M 13) & Rey et al. (2001) & $0.01 \div 0.03$ & $-1.65 \pm 0.06$ & -1.54 & $-1.39 \pm 0.06$ & 0.28 & $6.3 ; 8.1 ; 11.4 \times 10^{-4}$ \\
\hline
\end{tabular}

updated approach to the difficult problem of stellar modeling (see e.g., Castellani et al. 2001).

\section{Model tests and calibrations}

The theoretical background and the input physics adopted in our code have been discussed in Paper I (see also Ciacio et al. 1997, and Cassisi et al. 1998) and are listed in Appendix A. We only point out here that our models include atomic diffusion, including the effects of gravitational settling, and thermal diffusion with diffusion coefficients given by Thoul et al. (1994) while radiative acceleration (see e.g., Richer et al. 1998; Richard et al. 2002) is not taken into account. For convective mixing, we adopt the Schwarzschild criterion to define regions in which convection elements are accelerated (see the description in Brocato \& Castellani 1993). It is known that diffusion is a slow process; for masses of about $3 M_{\odot}$ or higher (that is for clusters of ages lower that about $350 \mathrm{Myr}$ ) its influence is negligible. Effects of rotation (see e.g., Maeder \& Zahn 1998; Palacios et al. 2003) are not included in our models.

As in previous papers, to assess the adequacy of the predicted evolutionary scenario we follow the procedure of submitting stellar models to a preliminary test to calibrate the mixing length parameter governing the efficiency of convection at the surface of a stellar structure and, thus, the predicted effective temperature of stars with convective envelopes.

Following this procedure, our models have been satisfactorily tested relative to Solar Standard Models (SSM; see e.g., Degl'Innocenti et al. 1997), young metal-rich galactic clusters with Hipparcos parallaxes (Castellani et al. 2001; Castellani et al. 2002) and moderately metal-rich galactic globulars (Paper I). We have adopted - in all cases - a ratio $\alpha$ between the mixing length and the local pressure scale height of $\alpha=l / H_{p} \sim 1.9$. Here we note that SSM requires element diffusion, so the calibration of $\alpha$ with solar models without diffusion does not appear meaningful. The same models also provided a satisfactory fit for young globulars in the LMC (Brocato et al. 2003).

For metal-poor stars, one finds in the Galactic globulars the natural candidates for a further test. In Cassisi et al. (1999) we fitted our low mass stellar models to globular clusters adopting $\alpha \sim 2.0$ for metallicities $Z \sim 10^{-4}$, but $\alpha \sim 2.3$ for $Z \sim 10^{-3}$. As we will discuss in this section, the increased accuracy of available CM diagrams allows us to submit the above calibration to even more stringent tests, investigating in particular the disturbing evidence for the need for a larger mixing length only for clusters with a metallicity of about $Z \sim 10^{-3}$. To this purpose we searched in literature for well observed, not too heavily reddened globular clusters for which good $(V, B-V)$ diagrams are available and with $[\mathrm{Fe} / \mathrm{H}]$ values in the range explored in this paper. We consequently selected (as in Cassisi et al. 1999) M 68 as a suitable sample of the most metal-poor clusters, but now adding the very accurate CM diagrams recently made available by Rey et al. (2001) for the moderately metal-rich clusters M 3 and M 13. Table 1 lists the three selected clusters together with the source of the photometric data, the range of values for the reddening available in the recent literature, current estimates for $[\mathrm{Fe} / \mathrm{H}]$ and for the amount of $\alpha$-enhancement.

As is well known, the effect of the $\alpha$-enhancement on the evolutionary tracks and isochrones of Population II stars can be simulated by using a scaled solar mixture corresponding to the actual total metallicity. Isochrones calculated with enhanced $\alpha$ elements are very well mimicked in all the main characteristics by the standard solar mixture isochrones of metallicity: $Z=Z_{0}\left(0.638 f_{\alpha}+0.362\right)$ where $f_{\alpha}=10^{[\alpha / \mathrm{Fe}]}$ and $Z_{0}$ is the initial (nonenhanced) metallicity (see e.g., Salaris et al. 1993; Salaris \& Weiss 1998). The last column in Table 1 gives these "equivalent $Z$ " as evaluated on the basis of $[\mathrm{Fe} / \mathrm{H}]$ values according to well known relations and by adopting the recent determination $[Z / X]_{\odot}=0.0230$ (see e.g., Bahcall et al. 2001).

Data in Table 1 show that cluster metallicities are in general far from being firm observational results. For the most metalpoor cluster, M 68, the uncertainty is still tolerable and the actual metallicity can be safely put around the value $Z=0.0002$. As already found, Fig. 1 shows that adopting this metallicity the best fit of the clusters requires $\alpha=2.0$ with an age of 11 Gyr. As in previous works, the fitting has been performed making use of the predicted HB luminosity to constrain the cluster distance modulus and, consequently, the cluster age. However, one may be reluctant to rely uncritically on such a theoretical prediction, since HB models are much more dependent on uncertaintes in the physical inputs than TO and subgiant branch (SGB) models. In this context evidence from the pulsational behavior of RR Lyrae variables in galactic globulars has already indicated that perhaps (i.e. if pulsational theory is taken as face value) our theoretical HB are too bright, even if by only $\approx 0.05 \mathrm{mag}$ (Marconi et al. 2003). The discrepancy increases for models without diffusion. However, Fig. 1 also shows that the calibration of $\alpha$ is not dramatically dependent on the assumed cluster distance modulus. Decreasing the distance modulus by $0.1 \mathrm{mag}$ to 15.20 one would derive a cluster age of $12 \mathrm{Gyr}$ with the same $\alpha$. Here we note that the distance modulus so derived for the cluster appears in good 

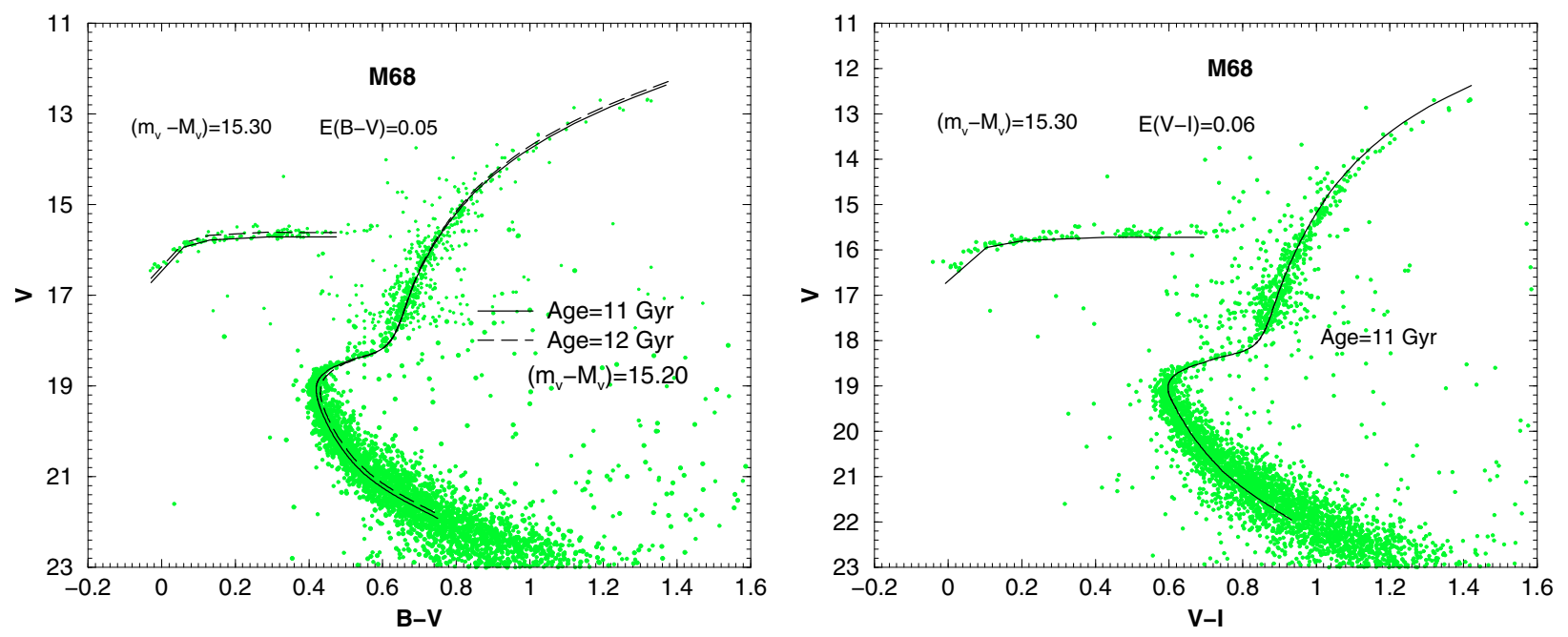

Fig. 1. The best fit of M 68 as obtained with the isochrone for $Z=0.0002,11 \mathrm{Gyr}$ and mixing length parameter $\alpha=2.0$ in $(V, B-V)$ (left panel) and in $(V, V-I)$ diagrams (right panel). In the left panel the fit with the $12 \mathrm{Gyr}$ isochrone and $\left(m_{v}-M_{v}\right)=15.20$ is also shown (dashed line). Color transformations are from Castelli (1999, see also Castelli et al. 1997).

agreement with the results by Carretta et al. (2000, but see also Gratton et al. 1997) who used Hipparcos parallaxes to obtain $\left(m_{v}-M_{v}\right)=15.25 \pm 0.06$.

To further discuss this calibration, we show in Fig. 2 a set of theoretical data displaying the behavior of theoretical isochrones with varying metallicity, age or $\alpha$. We find, for example, that increasing $\alpha$ from 2.0 to 2.3 decreases the extension in temperature of the SGB, the best fit requires shorter ages and also a larger distance modulus to balance the effect of the increased $\alpha$. Thus, we will keep $\alpha=2.0$ as a rather robust calibration of the mixing length in our models at $Z=0.0002$, bearing in mind that this calibration is supported by independent evaluations of the cluster apparent distance modulus. As a parallel check, we took advantage of the $(V, V-I)$ diagram presented by Walker (1994) for M 68 to further test the theoretical scenario. As shown in Fig. 1, one finds that the best fit obtained in the $(V, B-V)$ plane is preserved when passing to $(V, V-I)$, supporting the consistency of the adopted model atmospheres by Castelli (1999). When passing to larger metallicities, data in Table 1 show that, unfortunately, the uncertainties in the metallicity of the two calibrating clusters M 3 and M 13 are large, while the calibration depends sensitively on the assumption about this parameter. The only results on which all authors agree is that the metallicity of these two clusters should be very similar. Rey et al. (2001) showed that the subgiant branch in M 13 appears shorter than in M 3, in spite of the assumed common metallicity, reaching the conclusion that M 13 is older than M 3 by about 2 Gyr. A comparison between the Rey et al. data for the two clusters unambiguously reveals that- if both clusters have a common metallicity - M 3 has a larger reddening than $\mathrm{M} 13$ by about $\delta E(B-V) \approx 0.02 \mathrm{mag}$.

We find that adopting the Carretta \& Gratton metallicity value for the clusters requires that $\alpha$ cannot be constant when passing from $Z=0.0002$ to 0.001 (since for $\alpha=2.0$ one obtains a good fit for M 3 with $E(B-V)=0.00$, but the fit for M 13 requires a negative reddening). If one increases $\alpha$, the required reddening increases and Fig. 3 (left panel) shows that with the assumption $\alpha=2.3$ the TO and subgiant region of
M 13 are reasonably well fitted by the isochrone of $\approx 11.5 \mathrm{Gyr}$ with a distance modulus $\left(m_{v}-M_{v}\right) \approx 14.46$ and a reddening $E(B-V)=0.01 \div 0.02$. This stands in good agreement with Carretta et al. (2000: $\left.\left(m_{v}-M_{v}\right)=14.44 \pm 0.04\right)$. However, at larger luminosities, the cluster red giant branch (RGB) becomes progressively cooler than predicted. A similar effect has been discussed by Brocato et al. (1999) when fitting the RGB of NGC 6362 (whose metallicity is similar to both M 3 and M 13), again with $\alpha=2.3$. One may conclude that by adopting such a large mixing length for MS structures, one cannot avoid decreasing mixing length value along the RGB.

This result depends critically on the assumed metallicity. As shown in the lower panel of Fig. 2, decreasing the metallicity down to the Zinn \& West values makes the isochrone hotter, increasing the reddening requirements. In contrast, the SGB temperature range shrinks, requiring a shorter mixing length. As a result, the right panel of Fig. 3 shows that for $Z \approx 0.0006 \mathrm{M} 13$ can be reasonably well fitted assuming $\alpha=2.0$, with similar values of distance modulus and reddening, but increasing the age to about $13 \mathrm{Gyr}$. The isochrone now better reproduces the RGB shape. Thus metallicity plays a critical role in assessing the calibration of $\alpha$ for intermediate metallicity clusters, such as M 13 and M 3. Pending further and more precise constraints on this parameter, we prefer to adopt $\alpha=2.0$ throughout the explored range of metallicities, both to avoid a peculiar efficiency of superadiabatic convection at these metallicities and to better fit the RG branches. However, for the case $Z=0.001$ we present in the evolutionary library both cases ( $\alpha=2.0$ and $\alpha=2.3$ ), to allow a quantitative comparison of uncertainties in the fitting of observed CM diagrams.

Comparison of the two panels in Fig. 3 shows that the assumed value of $\alpha$ affects the evaluation of the cluster age, but has negligible impact on the derived distance moduli. We thus find that the best fit with the present isochrones appears able to produce distance moduli of comparable accuracy to those from the Hipparcos-based evaluations, independent of any uncertainties on the cluster metallicity. Rey et al. (2001) adopt $Z=0.0006$ to obtain for M 13 an age of $13 \mathrm{Gyr}$, in agreement 

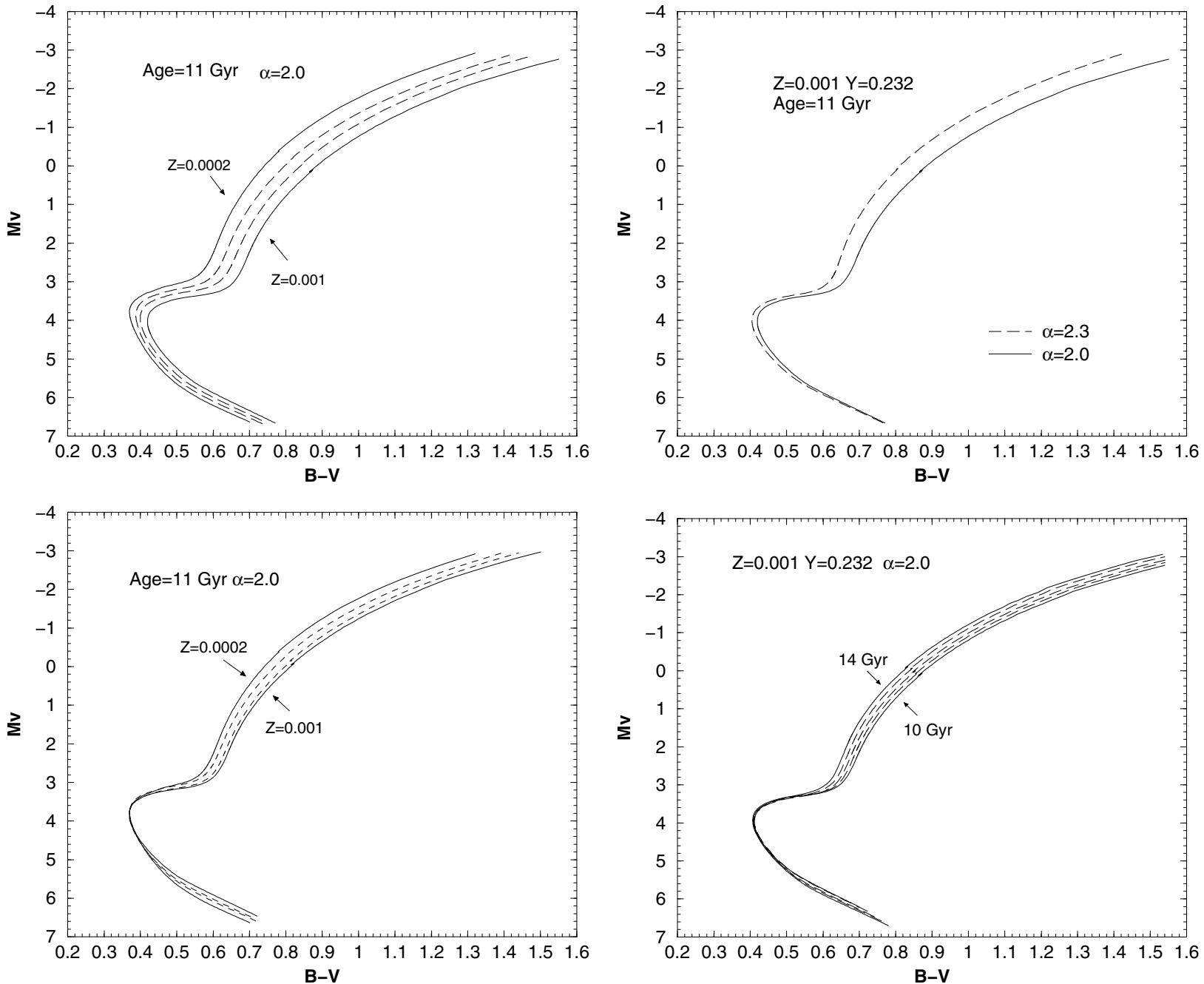

Fig. 2. Upper left panel: theoretical isochrones for $\alpha=2.0$, age $11 \mathrm{Gyr}$ and $Z=0.0002,0.0004,0.0006,0.001$. Upper right panel: theoretical isochrones for $Z=0.001$, age $11 \mathrm{Gyr}$, as computed under the two labeled assumptions about the mixing length. Bottom left panel: the isochrones as in the upper left panel but shifted to make TO.s to coincide each other; bottom right panel: isochrones for $Z=0.001$ and different ages shifted as in the left panel. Color transformations from Castelli (1999, see also Castelli et al. 1997).
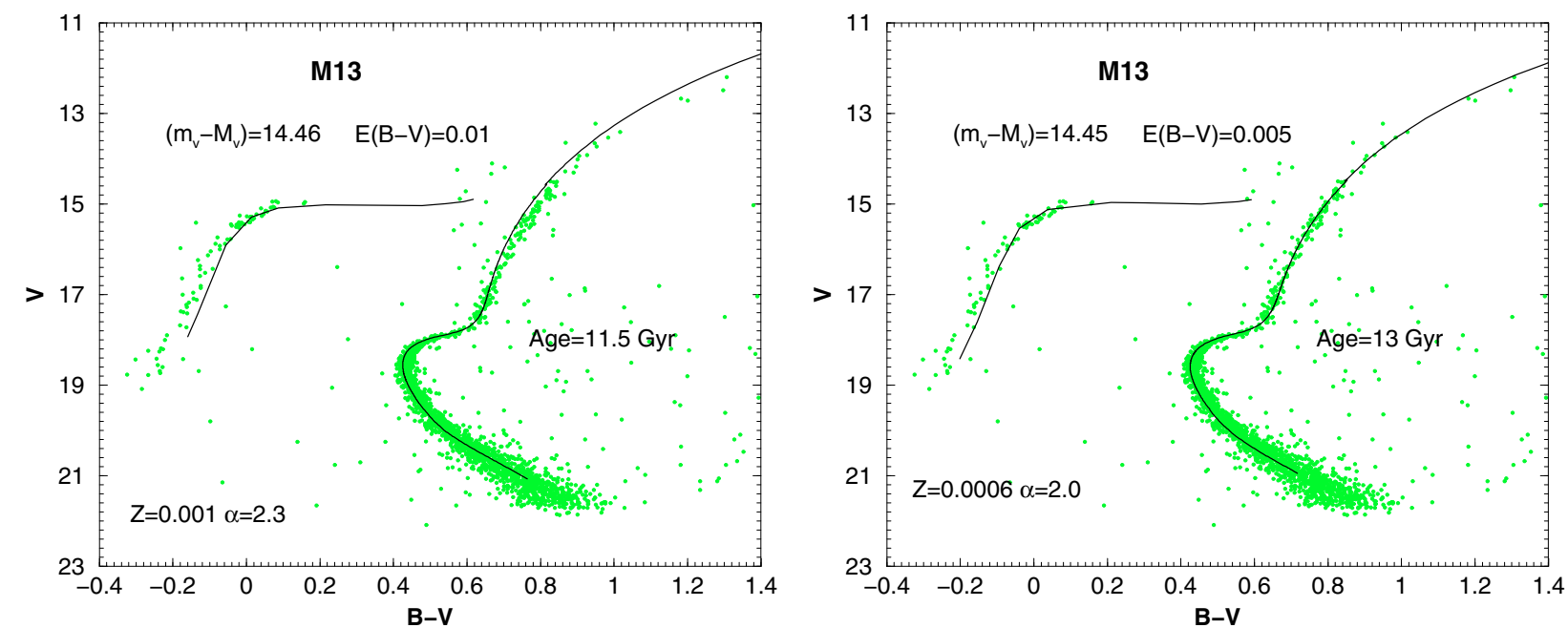

Fig. 3. Left panel: the best fit of M 13 as obtained with the isochrone for $Z=0.001,11.5 \mathrm{Gyr}$ and mixing length parameter $\alpha=2.3$. Right panel: the same but for $Z=0.0006,13 \mathrm{Gyr}$ and $\alpha=2.0$ (see text). 

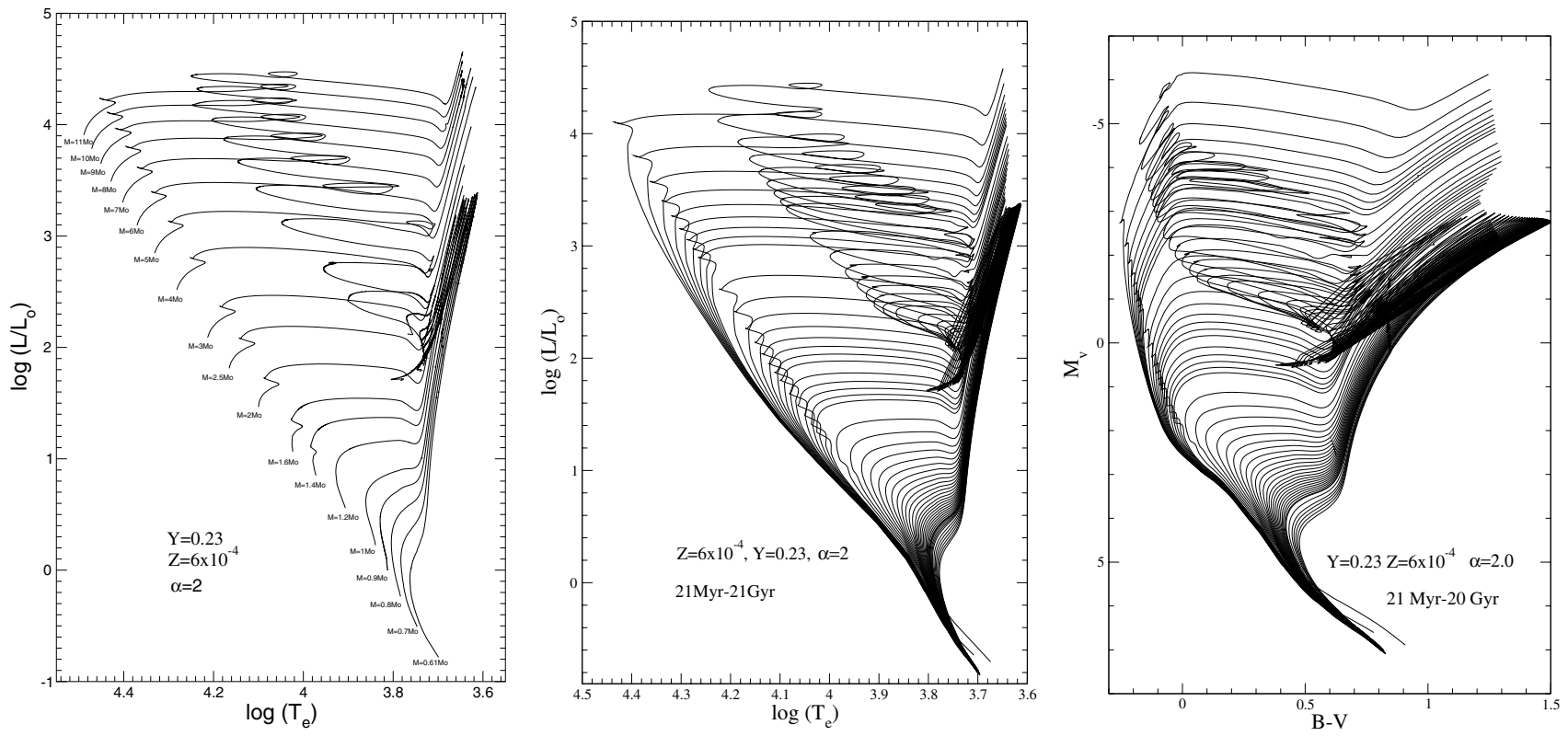

Fig. 4. Models for $Z=0.0006 Y=0.230 \alpha=2.0$. Left panel: evolutionary path in the $\left(\log \left(L / L_{\odot}\right), \log T_{\mathrm{e}}\right)$ diagram of the models computed for the labelled mass value. Middle panel: theoretical isochrones in the selected range of ages in the $\left(\log \left(L / L_{\odot}\right), \log T_{\mathrm{e}}\right)$ diagram. Right panel: theoretical isochrones in the selected range of ages in the $\left(M_{V}, B-V\right)$ diagram.

with our result. This is the consequence of the good agreement between present isochrones and those of Yi et al. (2001), as we will discuss in the next section and in the Appendix A.

\section{The models}

The computed evolutionary models cover with a fine grid the mass range 0.6 to $11 M_{\odot}$ for the adopted chemical compositions $Z=0.0002 Y=0.230, Z=0.0004 Y=0.230$, $Z=0.0006 Y=0.230, Z=0.001 Y=0.232$, where the amount of original helium has been evaluated by assuming a primordial helium abundance $Y_{P}=0.23$ and $\Delta Y / \Delta Z \sim 2.5$ (see e.g., Pagel \& Portinari 1998; Castellani et al. 1999). In all cases we assumed a solar mixture as given by Grevesse \& Noels (1993). All the models with evolutionary times smaller than the Hubble time have been followed from the Main Sequence through the $\mathrm{H}$ and He burning phases, until the $\mathrm{C}$ core ignition or the onset of thermal pulses in the advanced AGB phase. In the case of low mass stars undergoing violent He flashes, stellar structures at the Red Giant Branch (RGB) tip have been used to produce the corresponding Zero Age Horizontal Branch (ZAHB) models, further evolving these models until the onset of thermal pulses. Less massive stars $\left(M<0.7 M_{\odot}\right)$, whose evolutionary times are longer than the Hubble time, have been evolved up to central $\mathrm{H}$ exhaustion. To allow a proper evaluation of isochrones with ages lower than $\approx 150 \mathrm{Myr}$, the PreMain Sequence phase of selected low mass models has been included in the isochrones.

The evolutionary characteristics of the models have already been described in the literature, and they will not be discussed here. We only notice that the mass of the He core at the central helium ignition appears in good agreement with the results of Dominguez et al. (1999). On the basis of evolutionary tracks, cluster isochrones in the age range $\sim 20 \mathrm{Myr}$ to $\sim 20 \mathrm{Gyr}$ have been evaluated for the four choices about the cluster metallicity; for $Z=0.001$ models are calculated for two different $\alpha$ values: 2.0 and 2.3. Figure 5 shows, as an example, the HR diagram for $Z=0.0006 \alpha=2.0$; isochrones for the two metallicities are also shown in the $\left(M_{V}, B-V\right)$ plane.

Detailed tables for both tracks and isochrones are available at the URL http://astro.df.unipi.it/SAA/ PEL/ZO.html (and at the CDS). For each mass or for each isochronal age, these tables give the luminosity and effective temperature, followed by the visual magnitude and colors in the Johnson and infrared Cousins bands, as derived by adopting the model atmospheres by Castelli (1999). In addition one finds for the four adopted chemical compositions, tables with the distribution of ZAHB models as produced by a $0.8 M_{\odot}$ progenitor giving for the various HB masses the luminosity, temperature, $V$ magnitude and $B-V$ color, together with files reporting the HB and AGB evolution of all these models. This procedure gives ZAHB corresponding to ages in the range 10 to $14 \mathrm{Gyr}$, depending on the metallicity. As usual, the small dependence of these ZAHB luminosities on age can be neglected. A detailed investigation on the effect of age on ZAHB luminosities has been published by Cassisi \& Caputo (2002).

To permit a detailed comparison among different evolutionary computations, the website contains files listing the structural evolution of three selected models $(M=0.9,2.0$ and 4.0 $M_{\odot}$ ) for all the selected chemical compositions. For each mass, a file lists the sequence number of the model, its age, central abundance by mass of $\mathrm{H}$ or $\mathrm{He}$, luminosity, effective temperature, central temperature and density, the maximum off-center temperature and its location, the mass of the convective core, He core and convective envelope and, in the last four columns, the fraction of the total luminosity released by pp, CNO and He nuclear burning and by the gravothermal energy. 

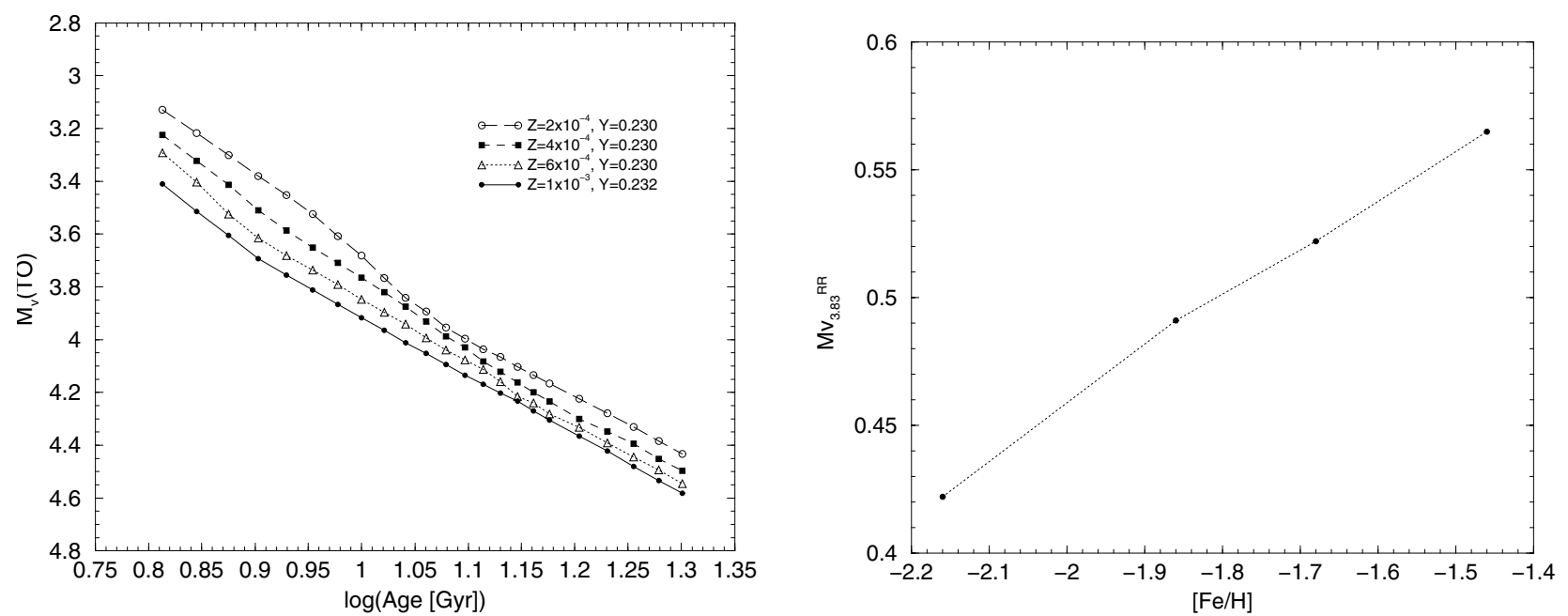

Fig. 5. Left panel: TO visual magnitude, $M_{V}(\mathrm{TO})$, as a function of the age, for the labeled chemical compositions. Right panel: ZAHB visual magnitude in the RR Lyrae region $\left(M v_{3.83}^{\mathrm{RR}}\right)$ as a function of $[\mathrm{Fe} / \mathrm{H}]$.

The evolutionary sequences for old clusters with ages greater than 6 Gyr closely follow theoretical predictions discussed in Cassisi et al. (1998, 1999). Here we take advantage of the finer grid of metallicities to present in Fig. 5, left panel, an improved calibration of the Turn Off magnitudes as a function of the cluster age covering the whole range $0.0002 \leq Z \leq$ 0.001. The right panel in Fig. 5 gives the calibration of the ZAHB magnitude taken at $\log T_{\mathrm{e}}=3.83$, i.e., near the central region of the RR Lyrae instability strip as a function of the cluster metallicity. A linear best fit through these data, taking into account an $\alpha$ enhancement of $[\alpha / \mathrm{Fe}] \approx 0.3$, gives:

$M_{V}(\mathrm{ZAHB})=0.20[\mathrm{Fe} / \mathrm{H}]+0.86$

a result well within current observational constraints (e.g., Chaboyer et al. 1998):

$M_{V}(\mathrm{ZAHB})=(0.23 \pm 0.04)[\mathrm{Fe} / \mathrm{H}]+(0.83 \pm 0.08)$

or by Carretta et al. (2000):

$M_{V}(\mathrm{ZAHB})=(0.18 \pm 0.09)[\mathrm{Fe} / \mathrm{H}]+(0.74 \pm 0.12)$.

Unfortunately, the large uncertainties in the empirical relations do not permit a solution to the problem of a possible overluminosity of present theoretical predictions.

As well known (see e.g., Stetson et al. 1996; Salaris \& Weiss 1997; Cassisi et al. 1998, 1999) TO and ZAHB magnitudes can be combined to calibrate the difference in magnitude $\left(\Delta M_{V}\right)$ between the Turn-Off (TO) and the helium burning HB phase as an age indicator, independently of both cluster distance and reddening. As discussed in Paper I, for ages $\leq 6 \mathrm{Gyr}$ the difference between the bright end of the main sequence (MT) and the bottom luminosity of the clump of He burning giants can provide an observational parameter that is able to derive from the CM diagram at least a rough estimate of the age independently on the cluster distance (see e.g., Castellani et al. 1999). Table 2 gives the $V$ magnitude of the main sequence termination (MT) and the difference $\left(\Delta M_{V}\right)$ in visual magnitude between the clump and MT as a function of the age in the range 150 Myr to 6 Gyr for all the metallicities investigated in this paper. MT and the clump magnitude have been evaluated at the maximum luminosity reached just after the overall contraction ( $\mathrm{H}$ exhaustion) and at the minimum luminosity of the He clump region, respectively. For each age, the original mass of stars populating the He burning clump is also shown. In the case of old globulars, our calibration gives ages in reasonable agreement with the ages recently determined by Salaris \& Weiss (2002). However, with respect to Salaris \& Weiss the present isochrone database has the additional advantage of including ages much lower than the typical globular clusters ages, down to about $20 \mathrm{Myr}$.

\section{Comparison with previous results}

A discussion of the differences between the present and Girardi et al. (2000) evolutionary models has already been given in Paper I (see also Castellani et al. 2000), whereas the existing differences between Girardi et al. and Yi et al. (2001) have been discussed in Gallart et al. (2003). Figure 6 shows a comparison between selected isochrones in the theoretical $\left(\log L / L_{\odot}, \log T_{\mathrm{e}}\right)$ plane for $Z=0.001$. A broader discussion on the main characteristics of the most updated extended sets of evolutionary models available in the literature can be found in Appendix A.

Inspection of data in Fig. 6 (left panel) shows that in the range of the largest ages (age $\approx 10 \mathrm{Gyr}$, mass at the TO $M \approx$ $0.85 M_{\odot}$ ) isochrones without element diffusion, e.g. in Girardi et al. (see also Salasnich et al. 2000), for any given TO luminosity give larger cluster ages and larger TO temperatures than diffusion isochrones. One finds a good agreement with the models of Yi et al. along the MS and SGB as a result of the quite similar input physics adopted to compute $\mathrm{H}$ burning models; the differences in temperatures in the upper portion of the RGB branch likely originate from different assumptions about the mixing length parameter $\alpha$.

Differences in temperatures between our models and Yi et al., both allowing for element diffusion, originate from different assumptions about the mixing length parameter $\alpha$, and 
Table 2. The $V$ magnitude of the main sequence termination (MT), the difference in luminosity between the clump and MT ( $\left.\Delta M_{V}\right)$ and the clump mass ( $\mathrm{m}$ in $M_{\odot}$ ) for isochrones with age $\leq 6 \mathrm{Gyr}$ (see text) and with the labeled chemical composition.

\begin{tabular}{|c|c|c|c|c|c|c|c|c|c|c|c|c|}
\hline Age & MT & $\Delta M_{V}$ & $m$ & MT & $\Delta M_{V}$ & $m$ & MT & $\Delta M_{V}$ & $m$ & MT & $\Delta M_{V}$ & $m$ \\
\hline & \multicolumn{3}{|c|}{$Z=0.0002 Y=0.23$} & \multicolumn{3}{|c|}{$Z=0.0004 Y=0.23$} & \multicolumn{3}{|c|}{$Z=0.0006 Y=0.23$} & \multicolumn{3}{|c|}{$Z=0.001 Y=0.232$} \\
\hline 0.15 & -1.924 & 0.074 & 3.55 & -1.622 & 0.389 & 3.59 & -1.628 & 0.359 & 3.63 & -1.673 & 0.268 & 3.67 \\
\hline 0.20 & -1.232 & 0.328 & 3.11 & -1.160 & 0.385 & 3.17 & -1.175 & 0.350 & 3.21 & -1.228 & 0.258 & 3.25 \\
\hline 0.25 & -0.834 & 0.378 & 2.83 & -0.809 & 0.385 & 2.88 & -0.824 & 0.349 & 2.91 & -0.882 & 0.256 & 2.90 \\
\hline 0.30 & -0.549 & 0.383 & 2.62 & -0.530 & 0.383 & 2.67 & -0.547 & 0.347 & 2.70 & -0.600 & 0.261 & 2.76 \\
\hline 0.35 & -0.382 & 0.332 & 2.47 & -0.294 & 0.385 & 2.51 & -0.312 & 0.345 & 2.54 & -0.354 & 0.271 & 2.59 \\
\hline 0.40 & -0.469 & 0.076 & 2.36 & -0.345 & 0.154 & 2.39 & -0.267 & 0.208 & 2.42 & -0.218 & 0.216 & 2.47 \\
\hline 0.45 & -0.550 & -0.154 & 2.27 & -0.389 & -0.049 & 2.30 & -0.301 & 0.012 & 2.32 & -0.223 & 0.043 & 2.36 \\
\hline 0.50 & -0.624 & -0.363 & 2.20 & -0.431 & -0.235 & 2.22 & -0.331 & -0.165 & 2.24 & -0.229 & -0.113 & 2.20 \\
\hline 0.60 & -0.712 & -0.687 & 2.07 & -0.508 & -0.564 & 2.08 & -0.383 & -0.473 & 2.10 & -0.241 & -0.389 & 2.12 \\
\hline 0.70 & -0.728 & -0.857 & 1.96 & -0.538 & -0.759 & 1.97 & -0.401 & -0.673 & 1.99 & -0.270 & -0.613 & 2.00 \\
\hline 0.80 & -0.640 & -0.903 & 1.88 & -0.475 & -0.836 & 1.89 & -0.346 & -0.769 & 1.90 & -0.223 & -0.721 & 1.90 \\
\hline 0.90 & -0.559 & -0.942 & 1.81 & -0.419 & -0.904 & 1.82 & -0.298 & -0.856 & 1.83 & -0.183 & -0.820 & 1.84 \\
\hline 1.00 & -0.485 & -0.976 & 1.74 & -0.368 & -0.965 & 1.76 & -0.254 & -0.933 & 1.76 & -0.148 & -0.912 & 1.78 \\
\hline 1.50 & -0.236 & -1.154 & 1.53 & -0.173 & -1.210 & 1.54 & -0.099 & -1.226 & 1.55 & -0.038 & -1.298 & 1.56 \\
\hline 2.00 & -0.121 & -1.354 & 1.40 & -0.078 & -1.441 & 1.41 & -0.017 & -1.452 & 1.41 & 0.017 & -1.528 & 1.42 \\
\hline 2.50 & -0.065 & -1.551 & 1.30 & -0.018 & -1.633 & 1.31 & 0.042 & -1.647 & 1.32 & 0.066 & -1.725 & 1.33 \\
\hline 3.00 & -0.020 & -1.712 & 1.23 & 0.031 & -1.785 & 1.24 & 0.090 & -1.802 & 1.24 & 0.106 & -1.888 & 1.25 \\
\hline 3.50 & 0.021 & -1.849 & 1.18 & 0.072 & -1.922 & 1.18 & 0.129 & -1.938 & 1.19 & 0.142 & -2.026 & 1.19 \\
\hline 4.00 & 0.058 & -1.956 & 1.13 & 0.111 & -2.043 & 1.13 & 0.156 & -2.059 & 1.14 & 0.163 & -2.151 & 1.15 \\
\hline 4.50 & 0.092 & -2.045 & 1.09 & 0.142 & -2.150 & 1.09 & 0.180 & -2.161 & 1.10 & 0.181 & -2.260 & 1.11 \\
\hline 5.00 & 0.121 & -2.169 & 1.05 & 0.170 & -2.239 & 1.06 & 0.201 & -2.251 & 1.06 & 0.198 & -2.355 & 1.00 \\
\hline 5.50 & 0.148 & -2.249 & 1.02 & 0.195 & -2.317 & 1.03 & 0.220 & -2.332 & 1.03 & 0.213 & -2.442 & 1.05 \\
\hline 6.00 & 0.172 & -2.316 & 1.00 & 0.218 & -2.383 & 1.00 & 0.238 & -2.411 & 1.01 & 0.226 & -2.522 & 1.02 \\
\hline
\end{tabular}
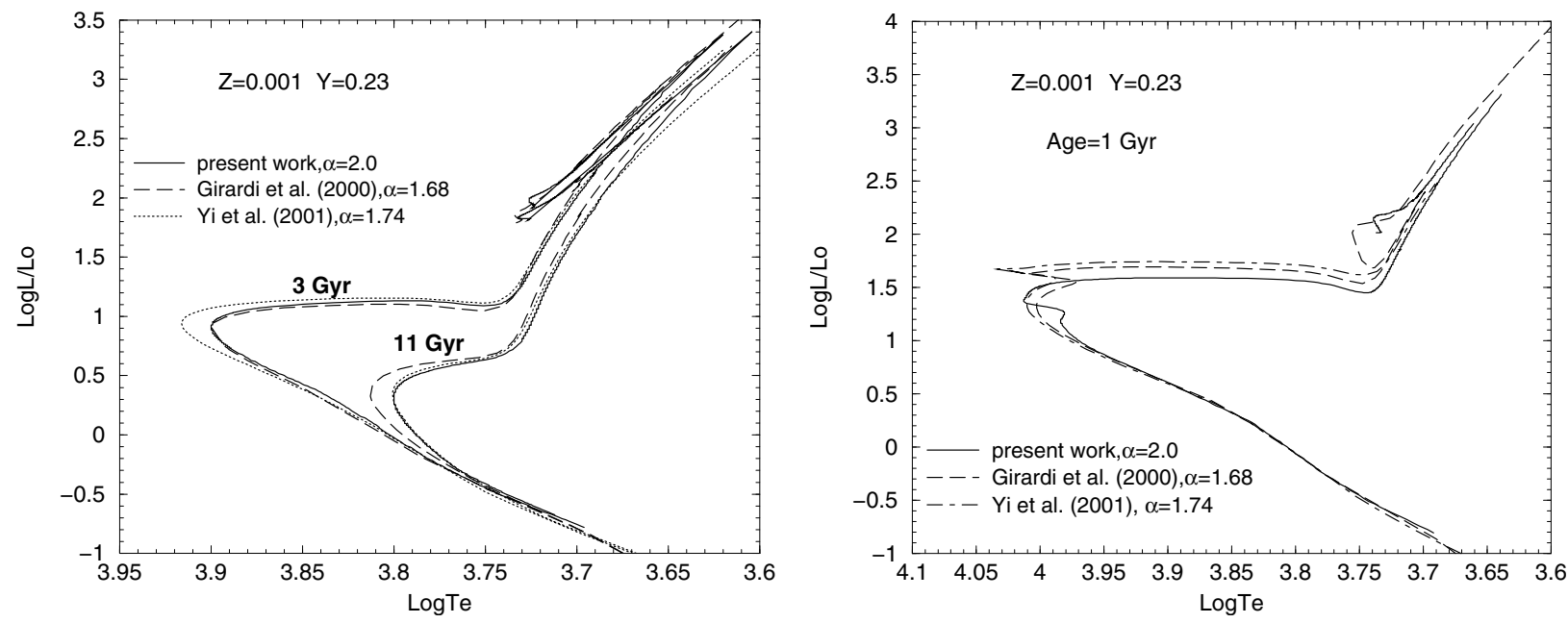

Fig. 6. Left panel: comparison between present, Girardi et al. (2000) and Yi et al. (2001) isochrones in the HR diagram for $Z=0.001 Y=0.23$ and for the two different labeled ages. Righ panel: as in the left panel but for age $=1 \mathrm{Gyr}$.

one finds that our large age isochrones with $\alpha=2.0$ agree well with the ones of Yi et al. along the MS and SGB as a result of the quite similar input physics adopted to compute $\mathrm{H}$ burning models.

For lower ages, one notes that the TO.s of the $3 \mathrm{Gyr}$ isochrones with (Girardi et al. 2000; Yi et al. 2001) or without (present computations) overshooting appear rather similar, with negligible differences between our and the Girardi et al. results. The reasons for this are twofold: the overshooting values adopted by $\mathrm{Yi}$ et al. and Girardi et al. are lower than those originally adopted in the Padua isochrones (see e.g.,
Bertelli et al. 1986), moreover for $1.0 \lessgtr M / M_{\odot} \lesssim 1.5$ the overshooting efficiency is further reduced, and for $M / M_{\odot} \leq 1.0$ the overshooting amount is set to zero to avoid the development of a small central convective zone in the solar model which would persist up to the present age of 4.6 Gyr. Note that the difference in TO temperature between models with and without overshooting does not scale as expected with the assumptions on $\alpha$, due perhaps to different treatments of the superadiabatic convection/inclusion of undershooting. As already discussed in Castellani et al. (2000) Girardi et al. models give fainter He burning clumps. We conclude that, due to the 
reduced extension of the convective cores of the stars populating clusters with $Z \approx 0.001$ and ages larger than or of the order of $3 \mathrm{Gyr}$, these clusters cannot provide any evidence for or against overshooting.

Evidence for efficient overshooting can however be found in younger clusters, with ages around $1 \mathrm{Gyr}$, as shown in the right panel of Fig. 6; one finds that at this age overshooting pushes the model beyond the Red Giant transition, and the overshooting signature will be represented by the reduced extension and population of the RG branch. We identify this prediction as the most robust test for overshooting, as already discussed in the seminal paper by Barbaro \& Pigatto (1984). However such a test is sensitively dependent on the cluster metallicity. A similar comparison of available isochrones reveals, for instance, that clusters at $Z=0.004$ with ages greater than about $1.5 \mathrm{Gyr}$, as the ones discussed in the recent paper by Woo et al. (2003), do not provide information about the overshooting efficiency.

\section{Final remarks}

In this paper we present and discuss canonical stellar evolutionary models produced for a grid of chemical compositions covering the range of Population II stars. For each given metallicity, we adopted a mixing length calibrated to reproduce the observed color of red giant branch in galactic globulars. On this basis we make available evolutionary tracks and cluster isochrones covering the range of ages $20 \mathrm{Myr}$ to $20 \mathrm{Gyr}$. The difference in magnitude between the top MS (the Blue Sequence) and He burning structures has been finally calibrated in terms of the cluster age for globular and open clusters. Due to the wide range in age, this calibration can be applied not only to population II clusters in the Galaxy but also to intermediate and young, low metallicity clusters in dwarf galaxies and in the Magellanic Clouds. VandenBerg et al. (1990) cautioned that uncertainties in input physics and/or model atmospheres make it unlikely that the predicted color difference between RG and TO is quantitatively correct. The discussion presented in this paper shows that a correct description of this parameter appears now within the theoretical possibilities as a result of the recent improvement in both input physics and atmospheric models.

Acknowledgements. We warmly thank Steve Shore and Pier Giorgio Prada Moroni for a careful reading of the manuscript and the anonymous referee for very useful comments. Financial support for this work was provided by the Ministero dell'Istruzione, dell'Università e della Ricerca (MIUR) under the scientific project "Stellar observables of cosmological relevance" (V. Castellani \& A. Tornambè, coordinators).

\section{Appendix A: Stellar evolutionary models}

Due to the relatively large number of stellar models available in the literature, we discuss the current situation. Theoretical predictions concerning stellar structures and their evolution depend on the adopted microphysics describing the behavior of the stellar plasma as well as on the assumptions about the efficiency of physical processes such as convection, microscopic diffusion, and overshooting. The comparison with observations, which is the final goal of evolutionary computations, requires a third ingredient: the model atmospheres needed to translate theoretical luminosities and effective temperatures into magnitudes and colors.

Current stellar models differ in a variety of choices concerning these inputs, producing small but significant differences in the results. Table A1 summarizes the main features of the sets of models (tracks and isochrones) available in the literature, which, to our knowledge, cover a wide range in chemical composition and stellar masses/isochrones ages. The quoted models obviously do not exhaust the rich and composite scenario of updated stellar evolutionary calculations; for example several authors calculated sets of tracks/isochrones for ancient populations or for very low mass stars (see e.g., Straniero et al. 1997; Chabrier \& Baraffe 1997; Cassisi et al. 2000; Vandenberg et al. 2000) or analyzed the characteristics of sets of intermediate mass stars (see e.g. Dominguez et al. 1999; Bono et al. 2000). In the table we add only models by VandenBerg et al. (2000) for low mass stars, as an example of evolutionary grids for old ages.

The table shows in the first three lines the range of masses, the range of metallicities $(Z)$ and the evolutionary phases covered by the quoted models. The following three lines give information on the treatment of the macroscopic mechanism, listing the adopted values of the mixing length parameter governing the efficiency of superadiabatic convection $\left(\alpha=l / H_{P}\right)$ and indicate whether or not diffusion and/or overshooting have been taken into account. The following five lines give the sources of the adopted physical inputs, listing in order the sources for the Equation of State (EOS), radiative opacity, cross sections for nuclear reactions, the evaluation of the electron conduction in degenerate matter and the production of cooling neutrinos. The adopted atmospheric models are listed in the last row. Asterisks in the table mark the data that require some additional information.

Present models: computations presented in this paper for the metallicity range $Z=0.0002$ to 0.001 do not include overshooting. However, the optional occurrence of overshooting has been presented and discussed in Paper I for the cases $Z=0.004$ and 0.008. OPAL96 EOS (Rogers et al. 1996) and OPAL96 Radiative Opacity (Iglesias \& Rogers 1996) when needed have been implemented with an updated version of the EOS of Straniero (1988) and with low temperature opacities from Alexander \& Fergusson (1994), respectively. Reaction rates are from the NACRE collaboration (Angulo et al. 1999).

Yi et al:: computations with different choices about the overabundance of " $\alpha$ " elements $([\alpha / \mathrm{Fe}]=0.0,0.3,0.6)$ are presented. For the conductive opacity Hubbard \& Lampe (1969) is adopted for $\log \rho\left(\right.$ in $\left.\mathrm{g} / \mathrm{cm}^{3}\right) \leq 6.0$ and Canuto (1970) for $\log \rho\left(\right.$ in $\left.\mathrm{g} / \mathrm{cm}^{3}\right)>6$.0. Diffusion is taken into account only for He. Magnitudes and colors are available as calculated by adopting two different atmospheric models: either from an update of Green et al. (1987) or from Lejeune et al. (1998) and Westera et al. (1999).

Girardi et al:: $\alpha$ enhanced models are presented elsewhere (Salasnich et al. 2000) for some metallicity values. The original Padua EOS has been implemented with MHD 
Table A.1. Comparison of the main characteristics of the most recent extended sets of evolutionary models available in the literature, see text.

\begin{tabular}{lccccc}
\hline \hline Models & Present & Yi et al. (2001) & Girardi et al. (2000) & Geneva & VandenBerg et al. (2000) \\
\hline Mass range $\left[M_{\odot}\right]$ & $0.6-11$ & $0.4-5$ & $0.15-7$ & $0.8-120$ & $0.5-1.0$ \\
Metallicity $(Z)$ & $2 \times 10^{-4} \div 8 \times 10^{-3}$ & $10^{-5} \div 8 \times 10^{-2}$ & $4 \times 10^{-4} \div 3 \times 10^{-2 *}$ & $10^{-3} \div 10^{-1}$ & $10^{-4} \div 3 \times 10^{-2 *}$ \\
Evo. phases & $\mathrm{H}+\mathrm{He}$ & $\mathrm{H}$ & $\mathrm{H}+\mathrm{He}$ & $\mathrm{H}+\mathrm{He}$ & $\mathrm{H}+\mathrm{He}$ \\
\hline$\alpha$ & $1.9-2.3$ & 1.74 & 1.68 & 1.6 & $\mathrm{NO}$ \\
Diffusion & Thoul et al. (1994) & Thoul et al. (1994)* & $\mathrm{NO}$ & NO* \\
Overshooting & NO* & $0.2 \mathrm{Hp}$ & $0.25 \mathrm{Hp}$ & $0.20 \mathrm{Hp}$ & - \\
\hline EOS & OPAL96+Str. (1998)* & OPAL96+Yale & Padua+MHD & Geneva* & Victoria \\
Rad. opacity & OPAL96+Alex. (1994)* & OPAL96+Alex. (1994) & OPAL92+Alex. (1994) & OPAL92+Kur. (1991) & OPAL96+Alex. (1994) \\
Nucl. reactions & NACRE* & Bahcall \& P. (1992) & Caug. \& Fow. (1988) & Caug. \& Fow. (1988) & Bahcall \& P. (1992) \\
El. conduction & Itoh et al. (1983) & Hubb. \& Lampe (1969)* & Hubb. \& Lampe (1969) & Hubb. \& Lampe (1969) & Hubb. \& Lampe (1969) \\
Neutrinos & Itoh et al. (1996) & Itoh et al. (1989) & Munakata et al. (1985) & Itoh et al. (1989) & Itoh et al. (1996) \\
\hline Model atm. & Castelli et al. (1999) & Lejeune et al. (1999)* & Castelli et al. (1999)* & Lejeune et al. (1999) & Vand. \& Clem (2003)* \\
\hline & & & & &
\end{tabular}

(Mihalas et al. 1990 and references therein) for temperatures lower than $10^{7} \mathrm{~K}$. For a critical analysis of the adopted color transformations in several photometric systems see Girardi et al. (2002).

Geneva: we refer to a series of papers from Schaller et al. (1992) to Mowlavi et al. (1998).

VandenBerg et al.: in more recent models (VandenBerg et al. 2002) both gravitational settling and radiative acceleration are taken into account. We quoted VandenBerg et al. (2000) because in VandenBerg et al. (2002) HB models are not calculated. The original helium abundance is assumed following the formula: $Y=0.235+2 Z$. Computations for models with an overabundance of " $\alpha$ " elements $([\alpha / \mathrm{Fe}]=0.3,0.6)$ are presented too. The color transformations adopted in the paper are quoted as "VandenBerg 2000, in preparation"; VandenBerg \& Clem (2003) is probably the correct reference for the published paper.

\section{References}

Alexander, D. R., \& Ferguson, J. W. 1994, ApJ, 437, 879

Angulo, C., Arnould, M., Rayet, M., et al. (NACRE Collaboration) 1999, Nucl. Phys. A, 645, 3

Bahcall, J. N., Pinsonneault, M., \& Basu, S. 2001, ApJ, 555, 990

Barbaro, G., \& Pigatto, L. 1984, A\&A, 136, 355

Bertelli, G., Bressan, A., Chiosi, C., \& Angerer, K. 1986, A\&AS, 66, 191

Bertelli, G., Nasi, E., Girardi, L., et al. 2003, ApJ, 125, 770

Bono, G., Caputo, F., Cassisi, S., Marconi, M., et al. 2000, ApJ, 543, 955

Brocato, E., \& Castellani, V. 1993, ApJ, 410, 99

Brocato, E., Castellani, V., Raimondo, G., \& Walker, A. R. 1999, ApJ, 527,230

Brocato, E., Castellani, V., Di Carlo, E., Raimondo, G., \& Walker, A. R. 2003, AJ, 125, 3111

Canuto, V. 1970, ApJ, 159, 641

Carraro, G., Vallenari, A., Girardi, L., \& Richichi, A. 1999, A\&A, 343,825

Carretta, E., \& Gratton, R. 1997, A\&AS, 121, 95

Carretta, E., Gratton, R., Clementini, G., \& Fusi Pecci, F. 2000, ApJ, 533, 215

Cassisi, S., \& Caputo, F. 2002, MNRAS, 333, 825

Cassisi, S., Castellani, V., Degl'Innocenti, S., \& Weiss, A. 1998, A\&AS, 129, 267
Cassisi, S., Castellani, V., Degl'Innocenti, S., Salaris, M., \& Weiss, A. 1999, A\&AS, 134, 103

Cassisi, S., Castellani, V., Ciarcelluti, P., Piotto, G., \& Zoccali, M. 2000, MNRAS, 315, 679

Castellani, V., Chieffi, S., Tornambe, A., \& Pulone, L. 1985, ApJ, 296, 204

Castellani, V., \& Degl'Innocenti, S. 1999, A\&A, 344, 97

Castellani, V., Degl'Innocenti, S., \& Marconi, M. 1999, MNRAS, 303, 265

Castellani, V., Degl'Innocenti, S., Girardi, L., et al. 2000, A\&A, 354, 150

Castellani, V., Degl'Innocenti, S., \& Prada Moroni, P. G. 2001, MNRAS, 320, 66

Castellani, V., Degl'Innocenti, S., Prada Moroni, P. G., \& Tordiglione, V. 2002, MNRAS, 334, 193

Castellani, V., Degl'Innocenti, S., Marconi, M., Prada Moroni, P. G., \& Sestito, P. 2003, A\&A, 404, 645

Castelli, F., Gratton, R. G., \& Kurucz, R. L. 1997, A\&A, 318, 841

Castelli, F. 1999, A\&A, 346, 564

Caughlan, G. R., \& Fowler, W. A. 1988, Atom. Data Nucl. Data Tables, 40, 283

Chaboyer, B., Demarque, P., Kernan, P. J., \& Krauss, L. M. 1998, ApJ, 494, 96

Chaboyer, B., Green, E. M., \& Liebert, J. 1999, AJ, 117, 1360

Chaboyer, B. 2001, in Astrophysical Ages and Times Scales, ed. T. von Hippel, C. Simpson, \& N. Manset (San Francisco: Astronomical Society of the Pacific), ASP Conf. Ser., 245, 162

Chabrier, G., \& Baraffe, I. 1997, A\&A, 327, 1039

Ciacio, F., Degl'Innocenti, S., \& Ricci, B. 1997, A\&AS, 123, 449

Degl'Innocenti, S., Dziembowski, W. A., Fiorentini, G., \& Ricci, B. 1997, ApJ, 7, 77

Dominguez, I., Chieffi, A., Limongi, M., \& Straniero, O. 1999, ApJ, 524,226

Ferraro, F. R., Messineo, M., Fusi Pecci, F., et al. 1999, AJ, 118, 1738

Gallart, C., Zoccali, M., Bertelli, G., et al. 2003, AJ, 125, 742

Girardi, L., Bressan, A., Bertelli, G., \& Chiosi, C. 2000, A\&AS, 141, 371

Girardi, L., Bertelli, G., Bressan, A., et al. 2002, A\&A, 391, 195

Gratton, R., Fusi Pecci, F., Carretta, E., et al. 1997, ApJ, 491, 749

Green, E., Demarque, P., \& King, C. 1987, The Revised Yale Isochrones and Luminosity Functions (New Haven: Yale Univ. Obs.)

Grevesse, N., \& Noels, A. 1993 in Origin and Evolution of the elements, ed. N. Prantzos, E. Vangioni-Flam, \& M. Casse (Cambridge: Cambridge Univ. Press), 15

Haft, M., Raffelt, G., \& Weiss, A. 1994, ApJ, 425, 222 
Harris, W. E. 2003, updated version of Harris W. E. 1996, AJ, 112, 1487 at the URL http://physwww.physics.mcmaster.ca/

Hubbard, W. B., \& Lampe, M. 1969, ApJS, 163, 297

Iglesias, C. A., \& Rogers, F. J. 1996, ApJ, 464, 943

Itoh, N., Mitake, S., Iyetomi, H., \& Ichimaru, S. 1983, ApJ, 273, 774

Itoh, N., Adachi, T., Nakagawa, M., \& Kohyama, Y. 1989, ApJ, 339, 354

Itoh, N., Adachi, T., Nishikawa, A., \& Kohyama, Y. 1996, ApJ, 470, 1015

Kraft, R. P., Sneden, C., Langer, G. E., et al. 1995, AJ, 109, 2586

Kurucz, R. L. 1991, in Stellar atmospheres: beyond classical models, NATO ASI Ser. C 341, ed. L. Crivellari, I. Hubeny, \& D. G. Hummer

Lejeune, T., Cuisinier, F., \& Buser, R. 1998, A\&A, 130, 65

Maeder, A., \& Zahn, J. P. 1998, A\&A, 334, 1000

Marconi, M., Caputo, F., Di Criscienzo, M., \& Castellani, M. 2003, ApJ, in press [arXiv: astro-ph/0306356]

Mateo, M. 1998, ARA\&A, 36, 435

Mihalas, D., Hummer, D. G., Mihalas, B. W., \& Däppen, W. 1990, ApJ, 360, 300

Monelli, M., Pulone, L., Corsi, C. E., et al. 2003, AJ, 126, 218

Mowlavi, N., Schaerer, D., Meynet, G., et al. 1998, A\&AS 128, 471

Munakata, H., Kohyama, Y., \& Itoh, N. 1985, ApJ, 296, 197

Palacios, A., Talon, S., Charbonnel, C., \& Forestini, M. 2003, A\&A, 399,603

Pagel, B. E. J., \& Portinari, L. 1998, MNRAS, 298, 747

Richard, O., Michaud, G., Richer, J., et al. 2002, ApJ, 568, 979

Richer, J., Michaud, G., Rogers, F., et al. 1998, ApJ, 492, 833

Rood, R. T., Carretta, E., Paltrinieri, B., et al. 1999, ApJ, 523, 752

Rosenberg, A., Saviane, I., Piotto, G., \& Aparicio, A. 1999, 122, 3219 AJ, 118, 2306

Rey, S. C., Yoon, S. J., Lee, Y. W., Chaboyer, B., \& Sarajedini, A. 2001, AJ, 122, 3219

Rogers, F. J., \& Iglesias, C. A. 1992, ApJS, 79, 507
Rogers, F. J. 1994, in ed. G. Chabrier, \& E. L. Schatzman, The equation of state in astrophysics (Cambridge: Cambridge University Press), IAU Coll., 147, 16

Rogers, F. J., Swenson, F. J., \& Iglesias, C. A. 1996, ApJ, 456, 902

Rutledge, G. A., Hesser, J. E., \& Stetson, P. B. 1997, PASP, 109, 907

Salaris, M., Chieffi, S., \& Straniero, O. 1993, ApJ, 414, 580

Salaris, M., \& Cassisi, S. 1996, A\&A, 305, 858

Salaris, M., \& Weiss, A. 1997, A\&A, 327, 107

Salaris, M., \& Weiss, A. 1998, A\&A, 335, 943

Salaris, M., \& Weiss, A. 2002, A\&A, 388, 492

Salasnich, B., Girardi, L., Weiss, A., \& Chiosi, C. 2000, A\&A, 361, 1023

Schaller, G., Schaerer, D., Meynet, G., \& Maeder, A. 1992, A\&AS, 96, 269

Stetson, P. B., VandenBerg, D. A., \& Bolte, M. 1996, PASP, 108, 560

Straniero, O., Chieffi, S., \& Limongi, M. 1997, ApJ, 490, 425

Straniero, O. 1988, A\&AS, 76, 157

Suntzeff, N. B., Schommer, R. A., Olszewski, E. W., \& Walker, A. R. 1992, AJ, 104, 1743

Thoul, A., Bahcall, J., \& Loeb, A. 1994, ApJ, 421, 828

Yi, S., Demarque, P., Kim, Y.-C., et al. 2001, ApJ, 533, 670

Walker, A. R. 1994, AJ, 108, 555

VandenBergh, D. A., Bolte, M., \& Stetson, P. B. 1990, AJ, 100, 445

VandenBerg, D., Richard, O., Michaud, G., \& Richer, J. 2002, ApJ, 571,487

VandenBerg, D., \& Clem, J. 2003, AJ, 126, 778

Westera, P., Lejeune, T., \& Buser, R. 1999, in Spectrophotometric Dating of Stars and Galaxies, ed. I. Hubeny, S. Heap, \& R. Cornett, ASP Conf. Proc., 192, 203

Woo, J.-H., Gallart, C., Demarque, P., Yi, S., \& Zoccali, M. 2003, AJ, 125,754

Zinn, R., \& West, M. J. 1984, A\&AS, 55, 45

Zinn, R. 1985, ApJ, 293, 424 